\title{
ROLE OF CIRCULATING OXIDIZED LDL: A USEFUL DIAGNOSTIC RISK MARKER IN CARDIOVASCULAR DISEASE
}

\author{
Deepti Mandsorwale, Bindu Sharma, Munindra Pratap Singh
}

The aim. To assess levels of circulating plasma ox- $L D L$ in various subgroups with different CVD and their relationship
with oxidative stress markers, MDA, catalase, and traditional coronary disease risk factors.

Material and methods. A total of 215 subjects divided into four groups comprising 54 healthy controls, patients with the SAP were 52 persons, with the UAP 53 ones, and with the AMI there were 56 persons, respectively. Lipid profile parameters (TC, TG, HDL-C, LDL-C, and VLDL-C), plasma MDA, catalase were estimated by kit methods, TBARS method, and colorimetric assay respectively. Plasma Ox-LDL was estimation by competitive ELISA kit method (Mercodia) with the help of specific monoclonal antibody $m A b 4 E b$. Results were present as mean $\pm S D$, significance level at $p$ values $<0.05$ with Student's unpaired t-test. Data analysis was performed by software package SPSS version 17.0.

Results. It showed a highly significant $(p<0.001)$ correlation in SAP, UAP, and AMI except for age in the SAP subgroup, moderately significant $(p<0.01)$. Lipid profile except $H D L-C$ was found highly elevated $(p<0.001)$ in subgroups than in healthy controls. HDL-C was higher $(p<0.001)$ in controls with respect to patient subgroups. Comparison of oxidative stress markers (MDA and catalase) and ox-LDH in control with patient's subgroup indicates highly significant $(p<0.001)$ correlation. The correlation between SAP \& UAP was insignificant $(p<0.05)$, SAP with AMI was significant $(p<0.05)$, and UAP \& AMI were highly significant $(p<0.001)$. Large interquartile range in SAP subgroup suggesting scattered deviation in the mean value as compared to UAP and AMI showed in the box and whiskers plot and concluded that significantly elevated level of ox-LDL in SAP, UAP, and AMI subgroups indicate its diagnostic importance of CVD.

Conclusions. Study concluded that significantly elevated level of ox-LDL in SAP, UAP, and AMI subgroups indicate its diagnostic importance of CVD

Keyword: acute myocardial infarction (AMI), unstable angina (UA), stable angina pectoris (SAP), cardiovascular dis$\operatorname{order}(C V D)$

How to cite:

Mandsorwale, D., Sharma, B., Singh, M. P. (2021). Role of circulating oxidized LDL: a useful diagnostic risk marker in cardiovascular disease. ScienceRise: Medical Science, 3 (42), 04-08. doi: http://doi.org/10.15587/2519-4798.2021.232830

(C) The Author(s) 2021

This is an open access article under the Creative Commons CC BY license

\section{Introduction}

Major independent risk factors for coronary artery disease (CAD) are advancing age, elevated blood pressure, elevated serum total and LDL cholesterol level, low serum HDL cholesterol level, diabetes mellitus, and cigarette smoking. The Framingham heart study has elucidated the quantitative relationship between these risk factors and CAD [1]. It has shown that the major risk factors are additive in predictive power and along with these factors oxidative stress and oxidized low-density lipoprotein (ox-LDL) have been proposed to play an important role in the development of atherosclerosis [2, 3]. The ox-LDL is present in atherosclerotic lesions from humans and animals [4]. Also it has been detected in the plasma of coronary heart disease (CHD) patients. Thus, perhaps it plays a key role in the generation of inflammatory processes in atherosclerotic lesions of all stages [5].

However, the potential association of systemically measured ox-LDL with coronary heart disease (CHD) is still a matter of controversy. Although several studies that investigated the association between circulating levels of oxidative biomarkers and CHD yielded fairly strong associations, others were not able to demonstrate any meaningful relationship with CHD [2].

Thus, the aim of the present observational casecontrol study was to assess levels of circulating plasma ox-LDL in various subgroups of patients with different cardiovascular diseases i.e., UAP, SAP, and AMI and their relationship with oxidative stress markers, malondialdehyde (MDA), catalase, and traditional coronary disease risk factors.

\section{Materials and methods}

The present case-control study consist of total of 215 subjects during the period from January 2018 to December 2019 , divided into four groups i.e., healthy control $(n=54)$, established patients with stable angina pectoris (SAP, $n=52$ ), unstable angina pectoris (UAP, $n=53$ ), and patients with 
acute myocardial infarction (AMI, $n=56$ ). All recruited subjects were selected from series of consecutive outdoor and indoor patient in collaboration of the department of Biochemistry and General Medicine, Varun Arjun Medical College and Rohilkhand Hospital, Shahjahanpur. Overnight fasting blood samples, $4.0 \mathrm{ml}$ were collected in vials containing EDTA anticoagulant and transported immediately to the Clinical Biochemistry Laboratory, VAMC \& RH, Shahjahanpur, for further analysis.

Lipid profile parameters namely, total cholesterol (TC), triglycerides (TG), HDL-C, LDL-C, and VLDL-C were estimated in all the study subjects by commercially available kit methods. Plasma MDA was estimated by TBARS method and catalase was estimated by colorimetric assay. Further, Plasma concentrations of Ox-LDL were quantitated by commercially available competitive sandwich ELISA kit method (Mercodia) with the help of specific monoclonal antibody $\mathrm{mAb} 4 \mathrm{~Eb}$ [2]. It has been shown that ox-LDL remains stable in stored samples and that the aforementioned assay has good reproducibility. Evaluation of the cardiovascular disease was performed by experienced investigators blinded for study aim. Established CVD patients with deranged lipid profiles were selected for the study.

The study was approved by an institutional ethical committee (reference number: VAMC\&RH/JR/3247/ 2018 and date 04/01/2018), and informed consent was obtained from each subject in accordance with principles of the Helsinki declaration. The patient's demographic profile, socioeconomic status, behavioural risk factors (sedentary lifestyle, dietary habits), and disease risk factor histories were recorded. Patients with diabetes mellitus, renal diseases, respiratory diseases, thyroid disorders, acute infection, or any other systemic illness and on lipid-lowering drugs for the past three months were excluded from the study. Regular smokers and alcohol abusers were also excluded. None of the control subjects had clinical or laboratory evidence of any disease that might have affected the parameters to be measured.

Statistical analysis. Data analysis was performed by software package SPSS version 17.0. Results were shown as mean \pm SD. Continuous variables of demographical and baseline characteristics were assessed with the use of Student's unpaired " $t$ " test to compare the levels of both the ratios between the test and control group. All $p$-values that were $<0.05$ were considered significant. Comparative analysis of plasma ox-LDL control within the patient subgroups (SAP, UAP and AMI) was also performed by box and whiskers plot to check the skewness and deviations within the values.

\section{Research results}

A total of 215 subjects recruited in the study for analyzing the risk factors, comprising 54 healthy control (31 male, 23 female), patients with stable angina pectoris (SAP) 52 (28 male, 24 female), patients with unstable angina pectoris (UAP) 53 (33 male, 20 female) and patients with acute myocardial infarction (AMI), 56 (34 male, 22 female) respectively as shown in Table 1 .

The baseline characteristics in control and patient's sub-groups comprised of age, BMI, systolic and diastolic blood pressure (expressed as mean $\pm \mathrm{SD}$ ). Results highlight highly significant $(p<0.001)$ correlation in all the three patient subgroups namely SAP, UAP and AMI except for age in SAP subgroup where it is moderately significant $(\mathrm{p}<0.01)$ in comparison to control as in Table 2.

Demographical characteristics of control and patient subgroups

Table 1

\begin{tabular}{|c|c|c|c|c|c|}
\hline \multirow{2}{*}{$\begin{array}{c}\text { S. } \\
\text { No }\end{array}$} & \multirow{2}{*}{$\begin{array}{c}\text { Demographical } \\
\text { characters }\end{array}$} & \multirow{2}{*}{$\begin{array}{c}\text { Control } \\
(\mathrm{n}=54)\end{array}$} & \multicolumn{3}{|c|}{ Patient subgroups $(\mathrm{n}=161)$} \\
\cline { 4 - 6 } & Sex, male, $\mathrm{n}(\%)$ & $31(57.4 \%)$ & $28(53.84 \%)$ & $33(62.26 \%)$ & $34(60.7 \%)$ \\
\hline 1 & Diet (Non-veg), $\mathrm{n}(\%)$ & $28(51.8 \%)$ & $29(55.76 \%)$ & $31(58.49 \%)$ & $30(53.57 \%)$ \\
\hline 3 & Smoker, $\mathrm{n}(\%)$ & $18(33.3 \%)$ & $21(40.38 \%)$ & $20(37.73 \%)$ & $24(42.85 \%)$ \\
\hline 4 & Alc. Intake, $\mathrm{n}(\%)$ & $3(5.55 \%)$ & $6(11.53 \%)$ & $5(9.43 \%)$ & $8(14.28 \%)$ \\
\hline 5. & Family history of CVD, $\mathrm{n}(\%)$ & $21(38.8 \%)$ & $25(48.07 \%)$ & $32(60.37 \%)$ & $30(53.57 \%)$ \\
\hline 6. & &
\end{tabular}

Table 2

Demographical characteristics of control and patient subgroups

\begin{tabular}{|c|c|c|c|c|c|}
\hline \multirow{2}{*}{ S. n } & \multirow{2}{*}{$\begin{array}{c}\text { Baseline } \\
\text { characters }\end{array}$} & \multirow{2}{*}{$\begin{array}{l}\text { Control } \\
(n=54)\end{array}$} & \multicolumn{3}{|c|}{ Patient subgroups $(\mathrm{n}=161)$} \\
\hline & & & $\operatorname{SAP}(n=52)$ & UAP $(n=53)$ & $\operatorname{AMI}(n=56)$ \\
\hline 1. & Age (yrs.) & $41.31 \pm 8.13$ & $46.88 \pm 8.96^{* *}$ & $49.81 \pm 8.06^{* *}$ & $50.01 \pm 7.25 * *$ \\
\hline 2. & $\operatorname{BMI}\left(\mathrm{kg} / \mathrm{m}^{2}\right)$ & $23.54 \pm 1.29$ & $25.63 \pm 1.70 * * *$ & $26.07 \pm 1.45 * * *$ & $25.02 \pm 1.40 * * *$ \\
\hline 4. & SBP $(\mathrm{mmHg})$ & $112.66 \pm 9.06$ & $139.78 \pm 10.67 * * *$ & $135.05 \pm 7.67 * * *$ & $129.28 \pm 10.42 * * *$ \\
\hline 5. & DBP $(\mathrm{mmHg})$ & $79.88 \pm 6.58$ & $89.5 \pm 5.80 * * *$ & $89.52 \pm 5.68 * * *$ & $85.69 \pm 7.36^{* * *}$ \\
\hline
\end{tabular}

Value are expressed as mean $\pm S D$, ${ }^{* *} p$ value $<0.01$ is moderately significant, ${ }^{* * *} p$ value $<0.001$ is highly significant

Table 3 shows the clinical and laboratory characteristics of all the three patient sub-groups i.e., SAP, UAP \& AMI compared with age and sex-matched control subjects. Total cholesterol, LDL-C, VLDL-C, and triglycerides were found highly elevated $(p<0.001)$ in patient subgroups than in healthy controls. On the contrary, HDL-C was higher
( $p<0.001)$ in control with respect to patient subgroups. Comparison of oxidative stress markers in control with patient's subgroup indicates a highly significant $(\mathrm{p}<0.001)$ correlation with respect to the levels of MDA and catalase.

Analysis of the data showed significant elevation in the levels of Ox-LDL from control to patient sub- 
groups. The differences were found highly significant $(p<0.001)$, between control and all the three patient subgroups. Non-significant $(\mathrm{p}>0.05)$ correlation was found on comparison between SAP and UAP. A further signifi- cant correlation was observed on comparison between SAP with AMI $(\mathrm{p}<0.05)$ whereas a highly significant correlation was found between UAP and AMI subgroup $(\mathrm{p}<0.001)$ as shown in Table 3 .

Table 3

Level of biochemical parameters in control and in patient subgroups

\begin{tabular}{|c|c|c|c|c|c|}
\hline \multirow{2}{*}{$\begin{array}{c}\text { S. } \\
\text { No }\end{array}$} & \multirow{2}{*}{ Biochemical parameters } & \multirow{2}{*}{$\begin{array}{l}\text { Control } \\
(n=54)\end{array}$} & \multicolumn{3}{|c|}{ Patient subgroups $(\mathrm{n}=161)$} \\
\hline & & & SAP $(n=52)$ & $\operatorname{UAP}(n=53)$ & AMI $(n=56)$ \\
\hline 1 & $\begin{array}{l}\text { Total Cholesterol } \\
\text { (TC) }(\mathrm{mg} / \mathrm{dl})\end{array}$ & $185.20 \pm 10.28$ & $215.69 \pm 16.04 * * *$ & $212.03 \pm 12.85^{* * *}$ & $213.69 \pm 15.5^{* * *}$ \\
\hline 2 & HDL-C (mg/dl) & $42.47 \pm 4.68$ & $35.42 \pm 3.52 * * *$ & $39.17 \pm 3.38 * * *$ & $37.56 \pm 3.53 * * *$ \\
\hline 3 & VLDL-C (mg/dl) & $30.14 \pm 2.82$ & $37.34 \pm 4.33 * * *$ & $36.17 \pm 3.16^{* * *}$ & $36.51 \pm 2.98 * * *$ \\
\hline 4 & LDL-C (mg/dl) & $112.58 \pm 8.61$ & $142.92 \pm 14.94 * * *$ & $136.68 \pm 14.10^{* * *}$ & $139.61 \pm 15.38^{* * *}$ \\
\hline 5 & $\begin{array}{l}\text { Triglycerides (TG) } \\
\text { (mg/dl) }\end{array}$ & $150.72 \pm 14.10$ & $186.74 \pm 21.69 * * *$ & $180.87 \pm 15.8 * * *$ & $182.56 \pm 14.93 * * *$ \\
\hline 6 & $\begin{array}{l}\text { Lipid peroxidation } \\
(\text { MDA })(\mu \mathrm{mol} / \mathrm{L})\end{array}$ & $2.15 \pm 0.51$ & $6.35 \pm 0.769 * * *$ & $4.50 \pm 0.722 * * *$ & $4.87 \pm 0.77 * * *$ \\
\hline 7 & $\begin{array}{c}\text { Catalase (CAT) } \\
(\mu \mathrm{mol} / \mathrm{min} / \mathrm{gmHb} .)\end{array}$ & $129.59 \pm 6.01$ & $67.26 \pm 7.33^{* * *}$ & $61.97 \pm 8.60 * * *$ & $64.36 \pm 8.62 * * *$ \\
\hline 8 & $\begin{array}{l}\text { Oxidized-LDL } \\
(\mathrm{U} / \mathrm{L})\end{array}$ & $95.27 \pm 3.70$ & $104.5 \pm 4.37^{* * *}$ & $103.64 \pm 2.43 * * *$ & $105.92 \pm 2.96^{* * *}$ \\
\hline
\end{tabular}

Value are expressed as mean $\pm S D,{ }^{*} p$ value $>0.05$ non-significant, ${ }^{* *} p$ value $<0.01$ moderate significant,

$*_{* * *} p$ value $<0.001$ highly significant

According to the box and whiskers plot, the interquartile range in the SAP subgroup was found to be very large suggesting scattered deviation in the mean values as compared to UAP and AMI subgroup. Also longer lower whisker suggests the existence of the majority of individual mean values towards the lower levels in the SAP subgroup. Box plots of UAP and AMI subgroup showed right skewness suggesting the existence of mean values towards the higher side according to Fig. 1.

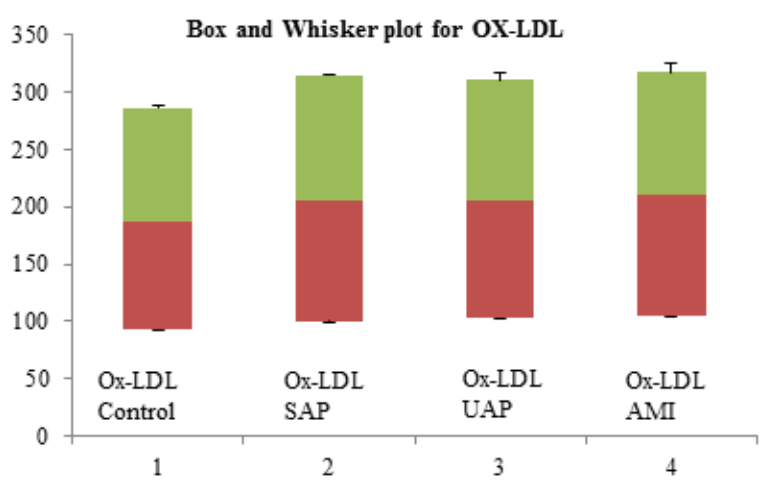

Fig. 1. Box and whisker plot for oxidized LDL

\section{Discussion of research results}

Cardiovascular disease (CVD) has emerged as the leading cause of death all over India, with coronary heart disease (CHD) affecting Indians at least 5-6 years earlier than their western counterparts [3].

CVD mortality and morbidity are promoted by major traditional risk factors such as hyperlipidaemia, hypertension, and smoking. The sequence of events leading to CVD includes endothelial dysfunction, atherosclerotic plaque formation, and rupture. Inflammation has been implicated in all these stages in the evolution of atherosclerotic plaques [6]. Moreover, oxidative stress is currently considered a key event in CVD development [4].

In the present case-control study, 215 subjects were selected for analyzing the risk factors in the various healthy control group $(n=54)$ and patient subgroup $(\mathrm{SAP}=52, \mathrm{UAP}=53$ and $\mathrm{AMI}=56)$, respectively. The baseline characteristics in control and patient subgroups such as age, BMI, systolic and diastolic blood pressure (expressed as mean \pm SD) and sex, smoking, diet, alcohol consumption, and family history (expressed as frequency distribution) were found elevated in SAP, UAP and AMI patient subgroup than the healthy controls as shown in Table 1.

In the present study, significant $(\mathrm{p}<0.05)$ correlations were found between the age of the control group in comparison to that of a patient subgroup. Similar, results were obtained earlier in studies conducted by Ndrepepa et al, 2008 [7]. This implies that the occurrence of CVD increases in older age i.e., oxidative stress accelerates the aging process as per Table 2 .

Highly significant $(p<0.001)$ correlation was found in terms of BMI when controls were compared with patient subgroups (Tab. 2) as supported by study performed by Shen et al 2017 [8]. Comparison between SAP Vs UAP has shown non-significant $(\mathrm{p}<0.05)$ correlation whereas a significant $(\mathrm{p}<0.001)$ correlation observed comparison between UAP and AMI subgroup. Thus, this suggests that increasing BMI must have a significant association with CVD.

Statistical analysis of systolic and diastolic BP between control and patient subgroups showed a highly significant correlation in all the three patient subgroups (SAP, UAP, and AMI) in comparison to control (Table 2). Similar results were obtained in the studies done by Heslop et al 2010 [9] and Schulz et al 2011 [10]. Also according to Van der Zwan et al, 2010 [11] the relationship between myeloperoxidase and blood pressure 
was strongest under conditions associated with oxidative stress, like obesity, low high-density lipoprotein cholesterol, metabolic syndrome, and type 2 diabetes. The strength of these associations was only marginally attenuated by adjustment for other cardiovascular risk factors.

The clinical and laboratory characteristics such as total cholesterol, LDL-C, VLDL-C, and triglycerides found highly elevated $(p<0.001)$ in inpatient subgroups than in healthy $(\mathrm{p}<0.001)$ in controls with respect to patient subgroups as shown in Tab. 3. Our findings correlate with the studies conducted by Shen et al 2017 [8], and Lobbes et al 2010 [12]. Furthermore, nonsignificance $(p>0.05)$ correlations were observed on comparisons within the patient's subgroups in above mentioned biochemical parameters i.e., TC, VLDL-C \& TG. Comparison of HDL-C indicates highly significant $(p<0.001)$ associations between SAP vs UAP, moderately significant $(\mathrm{p}<0.01)$ correlation between SAP vs AMI, and significant $(\mathrm{p}<0.05)$ an association was found between UAP and AMI as seen in studies performed by Linton et al, 2019 [6] as shown in Table 3.

According to NCEP guidelines, the optimal level for non-HDL-C should be $<130 \mathrm{mg} / \mathrm{dl}$. In the present study, the biochemical levels of non-HDL-C were found to be significantly elevated $(p<0.01)$ in all the three patient subgroups i.e., SAP, UAP, and AMI respectively in comparison to the control group as shown in Table 3.

The diagnostic and prognostic significance of non-HDL cholesterol is limited in different types of CVD subjects having only impaired lipid profile. Dyslipidaemia represents one of the most important risk factors for cardiovascular morbidity and mortality [13].

As in Table 3, comparison of control with the patient subgroups showed a highly significant $(p<0.001)$ correlation with respect to the level of MDA and Catalase, Karajibani et al, 2009 [14] revealed the differences in the levels of oxidant and antioxidant markers between CVD patients and healthy persons. A significant increase in MDA levels, a lipid peroxidant capacity indicates elevated oxidative stress in CVD patients. Evidences of ROS production leading to oxidative stress has been observed in atherosclerotic patients and also in patients suffering from hypercholesterolemia, hypertension, and diabetes mellitus [15].

The present study highlights a highly significant correlation $(p<0.001)$ with respect to ox-LDL in all the three patient sub-group-SAP, UAP, and AMI, respectively, in comparison to control. Furthermore, the comparison of UAP with AMI showed a highly significant $(\mathrm{p}<0.001)$ correlation whereas comparison of SAP with AMI indicates nearly significant $(p<0.08)$ correlation and that of SAP vs UAP showed insignificant association $(\mathrm{p}>0.05)$ as shown in Table 3. Moreover, according to box and whiskers plot in Fig. 1 observations of the mean \pm SD level of oxLDL in all the three patient subgroups indicate a larger deviation in the SAP subgroup in comparison to UAP and AMI subgroup. Thus, ox-LDL is used for the diagnosis of CVD, but its prognostic value is not reliable.

A number of case-control studies have examined the involvement of oxidative modifications of LDL in subjects with the presence of clinical cardiovascular disease [16]. Holvet et al [2] demonstrated elevated plasma concentrations of ox-LDL in patients with stable CHD or
ACSs compared with age-matched, apparently healthy controls. Also, ox-LDL concentrations were significantly higher in patients with MI than in patients with unstable or stable angina pectoris or age-matched controls subjects, suggesting a positive association between ox-LDL and the severity of ACSs. Findings from another study suggested that plasma levels of ox-LDL represent a more sensitive marker for the presence of CAD than the Global Risk Assessment Score, although a significant association between ox-LDL and most of the Framingham risk factors were observed [17].

The ox-LDL/Plasma cholesterol ratio was higher among cases compared with controls and also higher compared with hypercholesterolemic subjects free of an event, suggesting that the high plasma ox-LDL/TC ratio might serve as a possible indicator of increased risk of MI.

Recently, an association between metabolic syndrome and a high prevalence of ox-LDL has been found in a population-based cohort of individuals aged 70 to 79 years at baseline. In that study, participants with high ox-LDL concentrations had a greater risk of future CHD (relative risk $2.25 ; 95 \% \mathrm{CI}, 1.22$ to $4 . .15$ ), defined as coronary death or any hospitalization for MI, angina, coronary angioplasty, coronary artery bypass grafting, or chronic heart failure. However, in that study ox-LDL was not an independent predictor of incident CHD risk [18].

Limitations of the study. Our study deals only with Ox-LDL whereas several modern studies show that there are multiple modifications of LDL along with oxidation that allows the particle to develop its atherogenic property. Furthermore, the present study is conducted on a very small sample size that is not sufficient enough to justify the role of ox-LDL as a biomarker for CVD.

Prospects for further research. Our study along with several supporting evidence indicates that ox-LDL may exhibit both anti-inflammatory and pro-inflammatory properties. So, by elaborating its study regarding multiple modifications along with genetic polymorphism will probably leads to the development of good research/diagnostic tools for CVD and associated complications.

\section{Conclusion}

1. The usefulness of circulating oxidized LDL levels act as a diagnostic and prognostic marker in various subgroups of CVD patients i.e., SAP, UAP, and AMI according to the increasing severity of the disease.

2. Significantly elevated levels of ox-LDL in SAP, UAP \& AMI subgroup when compared with control subjects suggesting its diagnostic importance in CVD along with the elevated levels of traditional risk markers like oxidative stress (MDA \& catalase) and lipid profile as confounding factors.

3. It shows scattered deviation of ox-LDL levels in the SAP subgroup in comparison to control. UAP and AMI subgroup reflects its poor prognostic reliability in assessing the severity of the disease.

\section{Conflict of interests}

The authors declare that they have no conflicts of interest.

\section{Financing}

None 


\section{References}

1. Benjamin, E. J., Muntner, P., Alonso, A., Bittencourt, M. S., Callaway, C. W. et. al. (2019). Heart Disease and Stroke Statistics - 2019 Update: A Report From the American Heart Association. Circulation, 139 (10), e56-e528. doi: http://doi.org/10.1161/ cir.0000000000000659

2. Holvoet, P. (2012). Stress in Obesity and Associated Metabolic and Cardiovascular Disorders. Scientifica, 2012, 1-19. doi: http://doi.org/10.6064/2012/205027

3. Prabhakaran, D., Yusuf, S. (2010). Cardiovascular disease in India: learnt \& challenges ahead. Indian Journal of Medical Research, 132 (5), 529-530.

4. Yang, X., Li, Y., Li, Y., Ren, X., Zhang, X., Hu, D. et. al. (2017). Oxidative Stress-Mediated Atherosclerosis: Mechanisms and Therapies. Frontiers in Physiology, 8. doi: http://doi.org/10.3389/fphys.2017.00600

5. Di Pietro, N., Formoso, G., Pandolfi, A. (2016). Physiology and pathophysiology of oxLDL uptake by vascular wall cells in atherosclerosis. Vascular Pharmacology, 84, 1-7. doi: http://doi.org/10.1016/j.vph.2016.05.013

6. Linton, M. R. F., Yancey, P. G., Davies, S. S., Jerome, W. G., Linton, E. F., Song, W. L. et. al.; Feingold, K. R., Anawalt, B., Boyce, A., Chrousos, G., de Herder, W. W., Dungan, K. et. al. (Eds.. (2019). The role of lipids and lipoproteins in atherosclerosis. in Endotext. South Dartmouth: MD Text.com, Inc. Available at: https://www.ncbi.nlm.nih.gov/books/NBK343489/

7. Ndrepepa, G., Braun, S., Mehilli, J., Von Beckerath, N., Schömig, A., Kastrati, A. (2008). Myeloperoxidase level in patients with stable coronary artery disease and acute coronary syndromes. European Journal of Clinical Investigation, 38 (2), $90-96$. doi: http://doi.org/10.1111/j.1365-2362.2007.01908.x

8. Gao, S., Liu, J. (2017). Association between circulating oxidized low-density lipoprotein and atherosclerotic cardiovascular disease. Chronic Diseases and Translational Medicine, 3 (2), 89-94. doi: http://doi.org/10.1016/j.cdtm.2017.02.008

9. Heslop, C. L., Frohlich, J. J., Hill, J. S. (2010). Myeloperoxidase and C-Reactive Protein Have Combined Utility for LongTerm Prediction of Cardiovascular Mortality After Coronary Angiography. Journal of the American College of Cardiology, 55 (11), 1102-1109. doi: http://doi.org/10.1016/j.jacc.2009.11.050

10. Schulz, E., Gori, T., Münzel, T. (2011). Oxidative stress and endothelial dysfunction in hypertension. Hypertension Research, 34 (6), 665-673. doi: http://doi.org/10.1038/hr.2011.39

11. Van der Zwan, L. P., Scheffer, P. G., Dekker, J. M., Stehouwer, C. D. A., Heine, R. J., Teerlink, T. (2010). Hyperglycemia and Oxidative Stress Strengthen the Association Between Myeloperoxidase and Blood Pressure. Hypertension, 55 (6), $1366-$ 1372. doi: http://doi.org/10.1161/hypertensionaha.109.147231

12. Lobbes, M. B. I., Kooi, M. E., Lutgens, E., Ruiters, A. W., Lima Passos, V., Braat, S. H. J. G. et. al. (2010). Leukocyte Counts, Myeloperoxidase, and Pregnancy-Associated Plasma Protein A as Biomarkers for Cardiovascular Disease: Towards a MultiBiomarker Approach. International Journal of Vascular Medicine, 2010, 1-9. doi: http://doi.org/10.1155/2010/726207

13. Gibson, M. S., Domingues, N., Vieira, O. V. (2018). Lipid and Non-lipid Factors Affecting Macrophage Dysfunction and Inflammation in Atherosclerosis. Frontiers in Physiology, 9. doi: http://doi.org/10.3389/fphys.2018.00654

14. Karajibani, M., Hashemi, M., Montazerifar, F., Bolouri, A., Dikshit, M. (2009). The Status of Glutathione Peroxidase, Superoxide Dismutase, Vitamins A, C, E and Malondialdehyde in Patients with Cardiovascular Disease in Zahedan, Southeast Iran. Journal of Nutritional Science and Vitaminology, 55 (4), 309-316. doi: http://doi.org/10.3177/jnsv.55.309

15. Poznyak, A. V., Grechko, A. V., Orekhova, V. A., Chegodaev, Y. S., Wu, W.-K., Orekhov, A. N. (2020). Oxidative Stress and Antioxidants in Atherosclerosis Development and Treatment. Biology, 9 (3), 60. doi: http://doi.org/10.3390/biology9030060

16. Poznyak, A. V., Nikiforov, N. G., Markin, A. M., Kashirskikh, D. A., Myasoedova, V. A., Gerasimova, E. V., Orekhov, A. N. (2021). Overview of OxLDL and Its Impact on Cardiovascular Health: Focus on Atherosclerosis. Frontiers in Pharmacology, 11. doi: http://doi.org/10.3389/fphar.2020.613780

17. Zuliani, G., Morieri, M. L., Volpato, S., Vigna, G. B., Tch, C. B., Maggio, M. et. al. (2013). Determinants and clinical significance of plasma oxidized LDLs in older individuals. A 9 years follow-up study. Atherosclerosis, 226 (1), 201-207. doi: http://doi.org/10.1016/j.atherosclerosis.2012.10.028

18. Gómez, M., Vila, J., Elosua, R., Molina, L., Bruguera, J., Sala, J. et. al. (2014). Relationship of lipid oxidation with subclinical atherosclerosis and 10-year coronary events in general population. Atherosclerosis, 232 (1), 134-140. doi: http://doi.org/10.1016/ j.atherosclerosis.2013.10.026

Received date 11.03.2021

Accepted date 20.04.2021

Published date 31.05.2021

Deepti Mandsorwale, PhD, Assistant Professor, Department of Biochemistry, Shaikh-Ul-Hind Maulana Mahmood Hasan Medical College, Ambala Road, Saharanpur, India, 247001

E-mail: deepti16March@gmail.com

Bindu Sharma,' PhD, Associate Professor, Department of Biochemistry, Varun Arjun Medial College \& Rohilkhand Hospital, Lucknow Rd, District Shahjahanpur, Banthara, Uttar Pradesh, India, 242307

E-mail: drbindu2006@gmail.com

\section{Corresponding author}

Munindra Pratap Singh, MD, Demonstrator, Department of Physiology, Gajara Raja Medical College, Veer Savarkar Marg, Gwalior, Madhya Pradesh, India, 474009

E-mail: drmunindrapratapsingh@yahoo.com 